\title{
A Technology Mapping Algorithm for Heterogeneous FPGAs
}

\author{
Chi-Chou Kao and Yen-Tai Lai \\ Department of Electrical Engineering \\ National Cheng Kung University \\ Tainan, Taiwan
}

\begin{abstract}
In this paper, a technology mapping algorithm is proposed for heterogeneous FPGAs. The technology mapping problem is first formulated as a flow network problem. Then, an algorithm based on the min-cost max-flow algorithm is presented to select a proper set of feasible LUTs for various objectives. The objective, the total area composed of LUTs and routing area, are discussed in the paper. This algorithm has been tested on the MCNC benchmark circuits. Compared with other existing LUT-based FPGA mapping algorithms, the algorithm produces better characteristics.
\end{abstract}

\section{Introduction}

In a traditional lookup table (LUT)-based FPGA device, the configurable logic blocks (CLBs) are composed of $k$-input LUTs whose input number is constant. To maximize device utilization, heterogeneous FPGAs provide an array of homogeneous LUTs of different sizes or an array of physically heterogeneous LUTs. For example, the XC4000 [1] and ORCA2C [2] series FPGAs can be configured to have heterogeneous LUTs. The technology mapping problem for LUT-based FPGAs involves producing an equivalent circuit for a given circuit using only gates that can be implemented with LUTs.

This paper addresses the technology mapping problems for heterogeneous FPGA designs. There are several homogeneous FPGA technology-mapping algorithms for minimum layout size. However, most of these algorithms are unable to deal with heterogeneous FPGAs. A recent work [9] has shown that the area minimization-mapping problem for a tree network can be solved optimally in $O\left(n^{3}\right)$. However, this algorithm is significantly limited because the optimality holds only for the tree. The HeteroMap algorithm [10] was presented to reduce the number of LUTs for heterogeneous LUT-based FPGAs. An algorithm is proposed in this paper. The proposed algorithm can be configured for various objectives, the minimum number of LUTs, the total area, or others. To minimize the total area of a FPGA, two parts: 1) The LUT area and 2) the routing area are considered simultaneously.

The technology mapping problem is formulated first as a flow network problem. An algorithm based on the min-cost max-flow algorithm is then presented to select a proper set of LUTs from the set of feasible LUTs. An enumerating algorithm to generate all feasible LUTs is also presented. We implemented this algorithm and compared the empirical results with other LUT-based FPGA mapping algorithms. The results demonstrate the efficiency of this algorithm.

The remainder of this paper is organized as follows. The terminology and a graph-based formulation of the problem are described in Section 2. An algorithm for solving the problem is shown in Section 3. Section 4 gives an algorithm to generate the set of feasible cones. The objective, the total area, are discussed in Section 5. Experimental results are shown in Section 6. Our concluding remarks are presented in Section 7.

\section{Formulation of the Mapping Problem}

An FPGA technology mapping problem can be formulated as a graph based problem. A combinational logic circuit can be represented by a directed acyclic graph (DAG), $G=\left(V_{g} \cup V_{i o}, E\right)$. A vertex in $V_{g}$ represents a logic gate, while a vertex in $V_{i o}$ represents a pseudo gate that is either a primary input or a primary output. A directed edge $<i, j>$ exists in $E$ if the output of gate $i$ is the input of gate $j$. Notice that a primary input vertex has no in-coming edge and a primary output vertex has no out-going edge. Let $v$ and $u$ be two vertices of $V_{g}$. If $v$ is connected to $u$ by a single edge, $v$ is said to be a fan-in vertex of $u$ and $u$ is a fan-out vertex of $v$. Let $V_{s}$ be a subset of $V_{g}$ and $\overline{V_{s}}=V-V_{s}$. A fan-in signal of $V_{s}$ is a signal associated with an edge directed from a $\overline{V_{s}}$ vertex to a $V_{s}$ vertex. A fan-out signal of $V_{s}$ is a signal associated with an edge directed from a $V_{s}$ vertex to a $\overline{V_{s}}$ vertex. $\operatorname{Input}\left(V_{s}\right)$ is defined as representing the set of fan-in signals of $V_{s}$. Similarly, the Output $\left(V_{s}\right)$ represents the set of fan-out signals. A network is said to be $k$-bounded if the in-degree of every vertex is less than or equal to $k$ in the network.

Assume that $C_{v}$ is the subgraph induced by $V_{s}$. The subgraph $C_{v}=\left(V_{v}, E_{v}\right)$ is said to be a cone if vertex $v \in V_{v}$ exists such that for every vertex $u \in V_{v}$ there is a directed path from $u$ to $v$ in $C_{v}$. The vertex $v$ is called the tip of the cone. A cone, $C_{v}$, is said to be k-feasible if $\left|\operatorname{Output}\left(V_{v}\right)\right|=1$ and $\left|\operatorname{Input}\left(V_{v}\right)\right| \leqq k$. A vertex $u$ is said to be covered with $C_{v}$ if $u \in V_{v}$. Because $C_{v}$ is the induced subgraph of $V_{v}$, the set of fan-in signals of $C_{v}$ is exactly equal to the $\left|\operatorname{Input}\left(V_{v}\right)\right|$. For convenience, $\operatorname{Input}\left(V_{v}\right)$ and $\operatorname{Input}\left(C_{v}\right)$ are interchangeable in the remainder of this paper.

We assumed that a general LUT-based heterogeneous FPGA consists of LUTs of $n$ types. Each LUT of one type has $k$ inputs, $k \in\left\{k_{1}, k_{2}, \ldots, k_{n}\right\}$. Homogeneous FPGAs can be viewed as a special kind of heterogeneous FPGA with one and only one type of LUT. The technology mapping problem can be described as: Given a 2-bounded Boolean network, according to the objectives, find a set of feasible cones such that the union of all feasible cones includes all vertices.

Finding the maximum flow with minimum cost in a flow 
network can solve this problem. Given a directed acyclic graph, $G=\left(V_{g} \cup V_{i o}, E\right)$, let $V_{c}$ be the set of feasible cones in $G$. According to $G$, a flow network $G_{a s n}=\left(V_{g} \cup V_{c} \cup\{s, t\}, E_{b p} \cup E_{s t}\right) \quad$ is constructed in which:

1) $V_{g}$ is the set of vertices representing gates,

2) a vertex in $V_{c}$ represents a feasible cone,

3) $s$ and $t$ represent the source and the sink respectively,

4) there is an edge $e_{i j} \in E_{b p}$ directed from a vertex $i \in V_{g}$ to a vertex $j \in V_{c}$ if $i$ is a vertex in the $k$-feasible cone associated with $j$ and the capacity $\operatorname{cap}\left(e_{i j}\right)=1$,

$5)$ there is an edge $e_{s i} \in E_{s t}$ directed from $s$ to every vertex $i \in V_{g}$, and the capacity $\operatorname{cap}\left(e_{s i}\right)=1$, and

6) there is an edge $e_{j t} \in E_{s t}$ directed from each vertex $j \in V_{c}$ to $t$, and its capacity, $\operatorname{cap}\left(e_{j t}\right)$, is equal to the number of vertices in the cone $C_{j}=\left(V_{j}, E_{j}\right)$ associated with $j$.

Figure $1 . b$ illustrates a flow network constructed from the DAG shown in Figure 1.a. It is assumed that a LUT in the heterogeneous FPGA has $k$-inputs, $2 \leq k \leq 4$. In $G_{a s n}$, an edge is said to be saturated if the flow through it is equal to its capacity. Since a $V_{c}$ vertex has only one out-edge, for convenience, a $V_{c}$ vertex is also said to be saturated if its out edge is saturated. On the other hand, if the flow through a $V_{c}$ vertex is zero, the $V_{c}$ vertex is said to be empty.

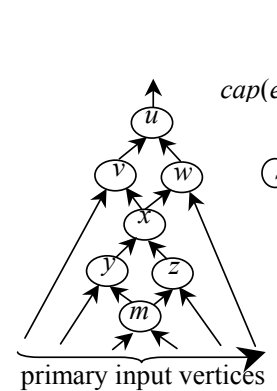

(a) vertices $V_{g} \operatorname{cap}\left(e_{i j}\right)=1$ feasible cones $V_{c}$
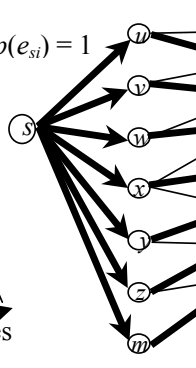

(b)
Fig. 1. a) A given DAG; b) The flow network constructed from $(a)$.

Theorem 1: A maximum flow in $G_{a s n}$ is equivalent to the solution of a technology mapping problem if the total flow is equal to $\left|V_{g}\right|$ and every $V_{c}$ vertex is either saturated or empty.

Proof: Since there is one and only one edge directed from $s$ to each $V_{g}$ vertex and its capacity is one, the upper bound of the total flow is the total number of vertices in $V_{g}$. If the maximum flow is equal to the total number of vertices in $V_{g}$, the mapping includes all of the vertices in $V_{g}$. If we select the set of feasible cones corresponding to the vertices, $V_{c}$ is saturated. By the construction rules, every gate is included in one and only one selected cone.

Consider the flow shown in Figure 1.b. The bold lines from a $V_{c}$ vertex to $t$ are saturated edges; the others are zero. Since the total flow is equal to $\left|V_{g}\right|$, by Theorem 1 , the set of cones induced by $\{u, v, w\}$ and $\{x, y, z, m\}$ can be selected to be an optimal mapping solution. There are several ways to generate a set of feasible cones [4]. If the set of feasible cones is not rich enough, it may not be possible to obtain a feasible solution. An algorithm to generate the set of feasible cones is presented in section 4 .

\section{An Algorithm for Finding the Min-cost Max-flow as Mapping Solution}

The mapping solution is not unique. Given an objective, we can define a cost function to assign a weight on each edge in $G_{a s n}$ and find the min-cost max-flow as the solution. There are several optimization objectives in the technology mapping process. Two objectives, the minimum number of LUTs and the total area, are discussed in Section 5.A and $B$. In this section, it is assumed the weight of cost on every edge is given.

There is an algorithm [13-14] that can find the maximum flow with minimum cost. However, a solution found by this algorithm does not ensure the flow through the sink edges is either saturated or zero. The bold lines in Figure 2 illustrate the min-cost max-flow network. It is seen that the flow through the vertex $a$ is neither saturated nor zero.

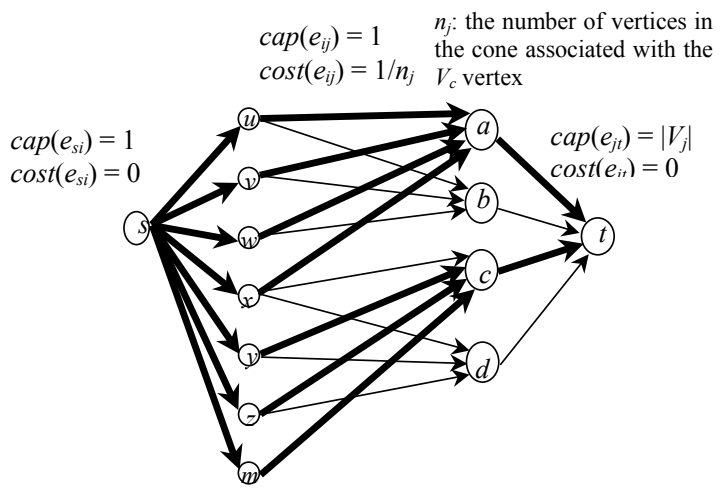

Fig. 2. A min-cost max-flow.

The flow path in $G_{a s n}$ found by the min-cost max-flow algorithm must be changed such that the $V_{c}$ vertex is either saturated or zero. When the flow path is changed, an algorithm which increases the minimum cost bound is needed. It has been proven that this problem is a NP-complete problem [13]. We will construct a max flow solution by a greedy algorithm. The strategy of this algorithm is as follows:

1) Find the minimum-cost maximum-flow in the $G_{a s n}$.

2) The cones associated with the saturated $V_{c}$ vertices are selected to be in the mapping solution.

3) A $V_{c}$ vertex, $j$, is called a candidate vertex if $j$ is non-saturated and the cone corresponding to $j$ includes no $V_{g}$ vertices covered by selected cones. The total cost increases if we change the path of flow through other non-saturated $V_{c}$ vertices such that $j$ becomes saturated. The increase of total cost to force $j$ saturated is denoted $\Delta C(j)$. For every candidate vertex, $j$, calculate $\Delta C(j)$.

4) According to the calculation in step 3, find the candidate vertex, $j$, whose $\Delta C(j)$ is minimum and change the path of flow to make $j$ be saturated.

5) Select the cone corresponding to $j$ to be in the mapping solution. If there exist candidate vertices, go to step 3 . 
6) If every $V_{g}$ vertex is covered, a mapping solution is obtained. Otherwise, no mapping solutions exist.

The min-cost max-flow algorithm [13-14] first finds a maximum flow in $G_{a s n}$. Then it constructs an auxiliary graph and iteratively reduces the total cost by finding directed cycles with negative costs in the auxiliary graph.

We need an approach to calculating $\Delta C(j)$ of a candidate vertex $j$. Let $C_{j}=\left(V_{j}, E_{j}\right)$ be the cone corresponding to $j$ and $V_{s}$ be a subset of $V_{j}$ such that in $G_{a s n}$ the flow coming from a $V_{s}$ vertex goes into $j$. Let $\overline{V_{s}}=V_{j}-V_{s}$ and $x \in \overline{V_{s}}$. Assume in $G_{a s n} e_{x j}$ is the edge directed from $x$ to $j$ and the flow from $x$ to a vertex $y$ passes along the edge $e_{x y}$. If the flow passing along $e_{x y}$ is changed to pass along $e_{x j}$, the increase of total cost is equal to $\operatorname{cost}\left(e_{x j}\right)-\operatorname{cost}\left(e_{x y}\right)$. Accordingly,

$$
\Delta C(j)=\sum_{x \in \bar{V}_{s}}\left(\cos \mathrm{t}\left(e_{x j}\right)-\cos \mathrm{t}\left(e_{x y}\right)\right)
$$

where $e_{x j}$ is the edge directed from $x$ to $j$ and $e_{x y}$ is the edge passed by the flow from $x$ to $y$.

Consider the example in Figure 2. It is seen that the vertex $c$ is saturated. Hence we select the cone induced by $\{x$, $y, z, m\}$ which is associated with $c$. The vertex $b$ is a candidate vertex. On the other hand, $d$ is not a candidate vertex because the cone associated with $d$ includes $\{x, y, z\}$ which are covered $V_{g}$ vertices. To make $b$ saturated, the flow along the three edges, $\langle u, a\rangle,\langle v, a\rangle$, and $\langle w, a>$, must be redirected to pass through $b$. To change the flow in each of these three edges, the cost increases $1 / 3-1 / 4=1 / 12$. Hence, $\Delta C(b)=1 / 4$. Since $b$ becomes saturated, the cone induced by $\{u, v, w\}$ is selected to cover the other vertices in the mapping solution.

\section{An Algorithm to Enumerate All Feasible Cones}

This section describes an algorithm to enumerate all feasible cones. Given a DAG, $G=\left(V_{g} \cup V_{i o}, E\right)$, we generate the feasible cones tipped at each vertex in $V_{g}$. Let $v \in V_{g}$ be a vertex and $C N(v)$ denote the set of cones $C_{v}$ tipped at $v$ and $\mid$ Input $\left(C_{v}\right) \mid \leq k$. Let Fin (v) be the set of fan-in vertices of $v$ and $\operatorname{Pcn}(v)=\bigcup_{v^{\prime} \in \operatorname{Fin}(v)} C N\left(v^{\prime}\right)$. Since a cone must be connected, a cone in $C N(v)$ must be the union of $\{v\}$ and a subset of $\operatorname{Pcn}(v)$. Therefore, we only need to inspect the cones in every $C N\left(v^{\prime}\right), v^{\prime} \in$ Fin $(v)$.

Accordingly, starting from every primary output vertex, all feasible cones can be found in an order of post-order traversal of $G$. Let $V_{v}$ be the union of $\{v\}$ and a subset of $\operatorname{Pcn}(v)$. The subgraph induced by $V_{v}$ is a $k$-feasible cone if and only if $\left|\operatorname{Input}\left(V_{v}\right)\right| \leq k$ and $\left|\operatorname{Output}\left(V_{v}\right)\right|=1$. To find $C N$ $(v)$, we must check the union of $\{v\}$ and every subset of $\operatorname{Pcn}(v)$.

The union of a subset of $P c n(v)$ can be equivalent to the union of another subset of $P c n(v)$. Assume $C_{x}$ and $C_{y}$ are two cones in $C N\left(v^{\prime}\right)$ and $C_{z}$ is the union $C_{x}$ and $C_{y}$. Every cone in $C N\left(v^{\prime}\right)$ includes $v^{\prime}$. If $C_{z}$ is a feasible cone, $C_{z}$ must include $v^{\prime}$ and $C_{z} \in C N\left(v^{\prime}\right)$. Otherwise, $C_{z}$ is not a feasible cone and $\left|\operatorname{Input}\left(C_{z}\right)\right|>k$. In this case, $\mid \operatorname{Input}(\{v\}$ $\left.\cup C_{z}\right) \mid>k$ and $\{v\} \cup C_{z}$ must not be a feasible cone. In other words, the union operation in $C N\left(v^{\prime}\right)$ is closure. Let a separation selection of $P c n(v)$ be a subset of $P c n(v)$ in which every member is selected separately from a $C N\left(v^{\prime}\right) \in$ $\operatorname{Pcn}(v), v^{\prime} \in$ Fin $(v)$. Therefore to find $C N\left(v^{\prime}\right)$, we can just check whether $\left|\operatorname{Input}\left(V_{s s}\right)\right| \leq k$ and $\left|\operatorname{Output}\left(V_{s s}\right)\right|=1$, where $V_{s s}$ is the union of $\{v\}$ and every separation selection of $\operatorname{Pcn}(v)$.

\section{The Optimization Objective}

The total area is the most important objective in the technology mapping process.

A large LUT must use a large area. A mapping with the minimum number of cones may not lead to the minimum total area because the size of the LUT grows exponentially proportional to the number of cone inputs. It is better to optimize the total area.

Assume that $C_{j}$ is a cone associated with a $V_{c}$ vertex and $A_{j}$ is the total area needed for using a logic block to implement $C_{j .}$. Similar to the cost function defined to minimize the total number of LUTs, if the cost of the edges between a $V_{g}$ vertex and the $V_{c}$ vertex is set to $\operatorname{cost}\left(e_{i j}\right)=$ $A_{j} / n_{j}$ and the cost of the other edges are zero, the total area can be minimized by finding the min-cost max-flow in $G_{a s n}$ where $n_{j}$ is the number vertices included in $C_{j}$.

Recall that the FPGA area includes the area for the LUTs and the area for routing. Let $A_{b}$ be the area for a logic block, and $A_{c}$ be the area needed for interconnection if a cone $C_{j}$ is selected. Then $A_{j}=A_{b}+A_{c}$. An approach for calculating $A_{b}$, and a method for estimating $A_{c}$ are needed.

To calculate $A_{b}$ the area for a LUT-based logic block, we used the logic block model shown in the literature 3 [1]. Let $B A$ be the bit area required to store a static RAM bit and $F A$ be the fixed area required to implement the $\mathrm{D}$ flip-flop and all of the other associated circuitry. The area for a logic block, $A_{b}$, is then:

$$
A_{b}=B A \times 2^{k}+F A \text {. }
$$

To estimate the routing area, we used the model proposed in the literature [3]. We can consider the needed routing area to be the space taken by the routing tracks on two of the four sides of the logic block, as shown in Figure 3.

Let $N_{p}$ be the number of pins. Assume that the pitch of a routing track is approximated as the square root of the area required by a bit. The dimension of a channel is then $N_{p} \times \sqrt{B A}$. The area for interconnection is:

$$
A_{c}=\left(N_{p}\right)^{2} \times B A+2 \sqrt{B A \times 2^{k}+F A} \times N_{p} \times \sqrt{B A} .
$$

According to (1) and (2), the total area of a logic block is: $A_{j}=\left\{B A \times 2^{k}+F A\right\}+\left\{\left(N_{p}\right)^{2} \times B A+2 \sqrt{B A \times 2^{k}+F A} \times N_{p} \times \sqrt{B A}\right\}$. 
According to the experimental results shown in [3], $N_{p}$ must be at least $k+1$ and proportional to the total number pins of CLBs.

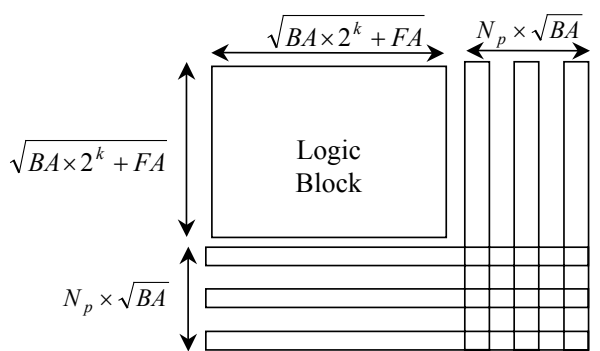

Fig. 3. A model for estimating the routing area.

IV Experimental Results

We used C language to implement the proposed algorithm on a SUN ULTRA SPARC workstation and tested several circuits from the MCNC logic synthesis benchmark set. To produce a more accurate area analysis for heterogeneous FPGA, testing was accomplished using FlowMap [5], HeteroMap [10], and the proposed algorithm on XC4000 series FPGAs that can implement circuits with 4-LUTs and 5-LUTs. Using 1.25 $\mu \mathrm{m}$ CMOS technology, $B A$ was estimated about $400 \mu \mathrm{m}^{2}$ and $F A$ was $5100 \mu \mathrm{m}^{2}$. Therefore, the total area of a 4-LUT was estimated as $\left\{400 \times 2^{4}+5100\right\}+\left\{(5)^{2} \times 400+2 \sqrt{400 \times 2^{4}+5100} \times 5 \times \sqrt{400}\right\}=$ $42948 \mu \mathrm{m}$. The results are shown in Table 1 . It confirms effectiveness of the proposed algorithm in terms of the area.

\begin{tabular}{|c|c|c|c|c|c|c|c|c|c|}
\hline \multirow[b]{2}{*}{ Circuits } & \multicolumn{3}{|c|}{ Proposed algorithm } & \multicolumn{3}{|c|}{ FlowMap } & \multicolumn{3}{|c|}{ HeteroMap } \\
\hline & $\#$ CLB & $\begin{array}{c}\text { Total } \\
\text { Area }(\mathrm{mm})\end{array}$ & CPU (s) & \# CLB & \begin{tabular}{|c|} 
Total \\
Area $(\mathrm{mm})$ \\
\end{tabular} & CPU (s) & \# CLB & \begin{tabular}{|c|} 
Total \\
Area $(\mathrm{mm})$
\end{tabular} & $\mathrm{CPU}(\mathrm{s})$ \\
\hline $5 \mathrm{xp} 1$ & 12 & 3095 & 5.6 & 20 & 5159 & 0.2 & 16 & 4127 & 0.2 \\
\hline 9sym & 44 & 11349 & 11.0 & 45 & 11607 & 0.3 & 48 & 12381 & 0.6 \\
\hline 9symml & 42 & 10833 & 15.4 & 47 & 12123 & 0.3 & 44 & 11349 & 0.6 \\
\hline C880 & 90 & 23214 & 55.3 & 183 & 47202 & 2.3 & 151 & 38948 & 13.3 \\
\hline alu2 & 77 & 19861 & 86.6 & 128 & 33016 & 1.5 & 114 & 29404 & 13.8 \\
\hline count & 31 & 7996 & 2.7 & 59 & 15218 & 0.4 & 51 & 13155 & 0.6 \\
\hline duke2 & 96 & 24762 & 27.5 & 140 & 36111 & 1.2 & 127 & 32758 & 2.3 \\
\hline $\mathrm{rd} 84$ & 25 & 6448 & 20.3 & 34 & 8770 & 0.4 & 31 & 7996 & 0.9 \\
\hline vg2 & 14 & 3611 & 1.0 & 31 & 7996 & 0.2 & 27 & 6964 & 0.3 \\
\hline Total & 431 & 111170 & 225.4 & 687 & 177201 & 6.8 & 609 & 157082 & 32.6 \\
\hline Comparison & & 1 & 1 & & $+59 \%$ & $-97 \%$ & & $41 \%$ & $-86 \%$ \\
\hline
\end{tabular}

Table I. Comparison of Area and CPU Time on XC4000 series FPGAs

\begin{tabular}{c||c|c|c|c|c|c|c|c|c}
\hline \multicolumn{1}{c||}{ Circuits } & \multicolumn{2}{c|}{ Proposed algorithm } & \multicolumn{3}{c|}{ FlowMap } & \multicolumn{3}{|c}{ HeteroMap } \\
\cline { 2 - 11 } & $\begin{array}{c}\text { Total } \\
\text { Area } \\
(\mathrm{mm})\end{array}$ & $\begin{array}{c}\text { Block } \\
\text { Area } \\
(\mathrm{mm})\end{array}$ & $\begin{array}{c}\text { Routing } \\
\text { Area } \\
(\mathrm{mm})\end{array}$ & $\begin{array}{c}\text { Total } \\
\text { Area } \\
(\mathrm{mm})\end{array}$ & $\begin{array}{c}\text { Block } \\
\text { Area } \\
(\mathrm{mm})\end{array}$ & $\begin{array}{c}\text { Routing } \\
\text { Area } \\
(\mathrm{mm})\end{array}$ & $\begin{array}{c}\text { Total } \\
\text { Area } \\
(\mathrm{mm})\end{array}$ & $\begin{array}{c}\text { Block } \\
\text { Area } \\
(\mathrm{mm})\end{array}$ & $\begin{array}{c}\text { Routing } \\
\text { Area } \\
(\mathrm{mm})\end{array}$ \\
\hline \hline 9sym & 2416 & 702 & 1714 & 3093 & 932 & 2161 & 3216 & 924 & 2292 \\
\hline 9 symml & 2628 & 772 & 1856 & 2967 & 888 & 2079 & 3171 & 926 & 2245 \\
\hline apex4 & 33720 & 9308 & 24413 & 51712 & 15380 & 36332 & 48881 & 13367 & 35514 \\
\hline alu2 & 7814 & 2195 & 5619 & 9722 & 2859 & 6862 & 9446 & 2632 & 6814 \\
\hline des & 37338 & 11157 & 26181 & 45448 & 13676 & 31772 & 46916 & 13722 & 33194 \\
\hline rd84 & 1487 & 416 & 1071 & 2596 & 752 & 1844 & 2341 & 635 & 1705 \\
\hline \hline Total & 85404 & 24550 & 60855 & 115537 & 34487 & 81050 & 113970 & 32206 & 81764 \\
\hline \hline Comparison & 1 & 1 & 1 & $+35 \%$ & $+40 \%$ & $+33 \%$ & $+33 \%$ & $+31 \%$ & $+34 \%$ \\
\hline
\end{tabular}

Table II. Comparison of Area on a Give Heterogeneous FPGA
A heterogeneous FPGA can consist of three or more types of LUTs. We select a heterogeneous FPGA architecture that consisted of three types of LUTs with input sizes of four, five, and six respectively. Table 2 shows the comparison results. Compared with FlowMap and HeteroMap, the proposed algorithm reduces $35 \%$ and $33 \%$ of the mapping area.

\section{References}

[1] Xilinx: "The programmable logic data book," Xilinx Inc., San Jose, CA, 1997.

[2] Lucent Technologies: "ORCA OR2C-A/OR2T-A series FPGAs data sheet," Lucent Technologies Inc., Allentown, PA, 1996.

[3] Rose, J. S., Francis, R. J., Lewis, D., and Chow, P.: "Architecture of programmable gate array: The effect of logic block functionality on area efficiency," IEEE Journal of Solid State Circuits, Vol. 25, No 5, Oct. 1990, pp. 1217-1225.

[4] Schlag, M., Kong, J., and Chan, P. K.: "Routability-driven technology mapping for lookup table-based FPGAs," IEEE Trans. on Computer-Aided Design of Integrated Circuits and System pp.13-26 Vol.13 No. 1, Jan. 1994.

[5] Cong, J., and Ding, Y.: "FlowMap: An optimal technology mapping algorithm for delay optimization in lookup-table based FPGA designs," IEEE Trans. on Computer-Aided Design of Integrated Circuits and System pp. 1-11 Vol.13 No. 1, Jan. 1994.

[6] Ahuja, R., Thomas, K., Magnanti, L., and Orlin, J. B.: "Network flows theory, algorithms, and applications," Prentice-Hall International Editions, 1993.

[7] Evans, J. R., and Minieka, E.: "Optimization algorithms for networks and graphs," Marcel Dekker, INC., 1992.

[8] He, J., and Rose, J.: "Technology mapping for heterogeneous FPGAs," presented at the ACM Int. Workshop FPGA, Feb. 1994.

[9] Korupolu, M. R., Lee, K. K., and Wong, D. F.: "Exact tree-based FPGA technology mapping for logic blocks with independent LUT," Proc, 35th ACM/IEEE Design Automation Conference, pp. 708-711, June 1998.

[10] Cong, J., and Xu, S.: "Delay-optimal technology mapping for FPGAs with heterogeneous LUT," UCLA Computer Science Dept. Tech. Report CSD-TR980015, 1998.

[11] J. Francis, J. Rose, and Z. Vranesic, “Chortle-crf: Fast Technology Mapping for Lookup Table-Based FPGAs," Proc, 28 ${ }^{\text {th }}$ ACM/IEEE Design Automation Conference, pp. 248-251, June 1991.

[12] J. Cong. and Y. Ding, "On area/depth trade-off in LUT-Based FPGA technology mapping," IEEE Transactions on VLSI Systems, pp. 137-148 Vol.2 No. 2, June 1994.

[13] M. R. Garey and D. S. Johnson, "Computers and Intractability: A Guide to the Theory of NP-Completeness," New York, W. H. Freeman, 1979. 